\title{
Extraoral photobiomodulation for prevention of oral and oropharyngeal mucositis in head and neck cancer patients: interim analysis of a randomized, double-blind, clinical trial
}

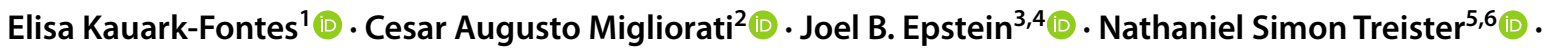 \\ Carolina Guimarães Bonfim Alves ${ }^{1,7}$ (1) $\cdot$ Karina Morais Faria ${ }^{7}$ (1) Natalia Rangel Palmier ${ }^{1,7}$ (1) . \\ Leticia Rodrigues-Oliveira' ${ }^{1}$ - Mariana de Pauli Paglioni ${ }^{1}$ (D) - Luiz Alcino Monteiro Gueiros ${ }^{8}$ (D) . \\ Karina G. M. da Conceição Vasconcelos ${ }^{9,10}$ (1) . Gilberto de Castro Jr ${ }^{11}$ (1) . Adriana Franco Paes Leme ${ }^{12}$ (D) .

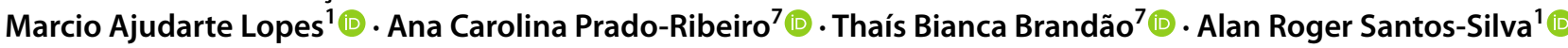

Received: 7 July 2021 / Accepted: 11 October 2021 / Published online: 28 October 2021

(c) The Author(s), under exclusive licence to Springer-Verlag GmbH Germany, part of Springer Nature 2021

\begin{abstract}
Purpose To assess the safety and efficacy of prophylactic extraoral photobiomodulation (PBM) for the prevention of oral and oropharyngeal mucositis $(\mathrm{OM})$ on clinical outcomes and survival in patients with oral cavity and oropharyngeal squamous cell carcinoma (OOPSCC).

Methods OOPSCC patients who received radiotherapy (RT) were prospectively randomized to two groups: prophylactic extraoral PBM and placebo. OM grade (NCI), pain (VAS), analgesia, and anti-inflammatory prescriptions were assessed weekly. Quality of life questionnaires (QoL) were performed at the first and last day of RT. Following RT, participants were evaluated quarterly for oncological outcomes follow-up.

Results Fifty-five patients met the inclusion criteria. The first occurrence of OM was observed at week 1, for the placebo group $(p=0.014)$. Later, OM onset and severity was observed for the PBM group, with first occurrence at week $2(p=0.009)$. No difference in severe OM incidence was observed $(p>0.05)$. Lower mean pain score was noted at week 7 for the PBM group (2.1) compared to placebo group (4.5) $(p=0.009)$. Less analgesics (week $3 ; p=0.009 /$ week $7 ; p=0.02$ ) and antiinflammatory prescription (week $5 ; p=0.0346$ ) were observed for the PBM group. Better QoL scores were observed for the PBM group at last day of RT $(p=0.0034)$. No difference in overall survival among groups was observed in 1 year of follow-up $(p=0.889)$.

Conclusion Prophylactic extraoral PBM can delay OM onset, reduce pain, and reduce analgesic and anti-inflammatory prescription requirements. Extraoral PBM was associated with better QoL. There was no evidence of PBM impact on oncological outcomes.
\end{abstract}

Trial registration TRN:RBR-4w4swx (date of registration: 01/20/2020).

Keywords Photobiomodulation · Oral mucositis · Radiotherapy $\cdot$ Quality of life $\cdot$ Overall survival

\section{Introduction}

Oral mucositis $(\mathrm{OM})$ is an acute side effect of the cytotoxic cancer treatment that is particularly severe in head and neck cancer (HNC) patients undergoing radiotherapy (RT) and chemoradiotherapy (CRT). OM often leads to debilitating

Alan Roger Santos-Silva

alanroger@fop.unicamp.br

Extended author information available on the last page of the article and dysfunction distress due to pain with impairment in eating, swallowing, and speech functions [36, 42]. This morbidity has marked negative impact on patient's quality of life (QoL); increases treatment costs due to the need of hospitalization, nutritional support, opioids use, antimicrobials and anti-inflammatory drugs; and may lead to new or prolonged hospitalization [24]. The incidence and severity of OM depend upon several risk factors associated with the oncological treatment and patient characteristics [27].

With level I scientific evidence, the Multinational Association of Supportive Care in Cancer/International Society 
of Oral Oncology (MASCC/ISOO) recommends the use of photobiomodulation (PBM) as an adjuvant intervention for prevention of OM in the HNC setting [43]. Although PBM is well established and accessible, there is great variability in PBM parameters, protocols, and equipment, which hampers consistent evaluation $[7,19]$. Another challenge to the large acceptance of PBM relies on the possibility that it may stimulate the growth of residual tumor cells or impact the field of cancerization in HNC [8, 17, 21, 37]. It is paramount that interventions used to mitigate $\mathrm{OM}$ do so without negatively impacting the effectiveness of the tumor treatment, especially in cases where the PBM application is anatomically adjacent to the tumor field, such as in HNC [39].

We conducted a randomized, double-blind clinical trial aimed to evaluate the effect of extraoral PBM prophylactic delivery on OM, OM-related pain, QoL, and cancer safety outcomes in oral and oropharynx squamous cell carcinoma (OOPSCC) patients during RT.

\section{Methods}

This double-blind, prospective clinical trial was conducted at Instituto do Cancer do Estado de São Paulo (ICESP), São Paulo, Brazil. Ethical approval was obtained from the National Human Research Ethics Committee (CAAE: $21,648,819.9 .0000 .5418)$. The study was conducted in accordance with the Declaration of Helsinki and reported according to the Consolidated Standards of Reporting Trials guidelines (CONSORT) [35]. The trial was registered in the International Clinical Trials Registry Platform (ICTRPWHO) and Brazilian Registry of Clinical Trials (ReBec) (Registration Number: RBR-4w4swx) [32]. We present the results of a planned interim analysis when at least 55 participants had completed a minimum of 1 year of follow-up. All participants included in the study provided informed consent.

\section{Patients}

Patients diagnosed with OOPSCC in stage III or IV (International Union Against Cancer, 8th edition) [9], over the age of 18 years, treated with curative RT protocols (60-70 Gy-2.0-2.12 Gy/day, 5 sessions/week) as a single modality or in association with CT were included. All included patients were submitted to the institutional standard-of-care dental treatment protocol before RT, designed to identify potential source of infection and maintain oral health such as complete oral prophylaxis, restorations, dental scaling/polishing, endodontic therapy, and tooth extraction if necessary [39]. Demographics and clinicopathological information were obtained from the electronic medical record system (Tasy, Java version; Koninklijke Philips N.V., 2004-2017).

Patients were excluded if they had distant metastasis, had previously received RT to the head and neck, or were scheduled to receive palliative RT.

Participants were blinded and randomly allocated into two groups: extraoral PBM and placebo. Two randomization lists, on blocks of 4 patients, were performed according to a 1:1 ratio. The lists were generated by SAS program (version 8.02). All patients received chlorhexidine $0.12 \%$ for daily use, verbal and written instructions about oral hygiene, abstinence from tobacco and alcohol, and risk of oral toxicities related to head and neck RT [23].

\section{PBM protocol}

Patients in the extraoral PBM group received daily prophylactic PBM for 5 consecutive days/week (Monday to Friday), from the first to the last day of RT. Two trained dentists administered the PBM by using the THOR LX2 unit with the red and near-infrared light emitting diode (LED) probe (THOR Photomedicine Ltd, Chesham, UK). The probe contains 69 diode LED, composed of $34 \times 660 \mathrm{~nm}$ (red; 10mW) and $35 \times 850 \mathrm{~nm}$ (near-infrared; $30 \mathrm{~mW}$ ) with a total power output of $1390 \mathrm{~mW}$, an outer diameter probe of $70 \mathrm{~mm}$, $63 \mathrm{~mm}$ of active area diameter, and an average power density of $44.6 \mathrm{~mW} / \mathrm{cm}^{2}$. The LED probe was applied flat against the patient's face and neck for $60 \mathrm{~s}$, at five treatment sites: right face side, central face on the lip area, left face side, cervical area on the left, and right sides $\left(50 \mathrm{~mW} / \mathrm{cm}^{2} \times 60 \mathrm{~s}=3.0 \mathrm{~J} /\right.$ $\mathrm{cm}^{2}$ per location) [40] (Fig. 1). The placebo/control PBM group underwent LED sham sessions with an inactivated extraoral probe, following the same model and daily applications as extraoral PBM group (Fig. 1). To ensure the blinding of participants, the extraoral sham sessions were performed with the same device, the activation button was pressed twice to simulate the application and activation sound (beep), and all participants wore dark safety googles. For safety and infection control purposes, a systematic disinfection routine with $70 \%$ alcohol ethylic was completed before and after each session; also, a disposable plastic film was used to cover the probe.

\section{Oral mucositis}

The same calibrated dentist, blinded to the allocation group, completed the clinical outcomes assessment, prior to the PBM session. All patients were evaluated weekly for the presence, topography, and severity of OM following the Common Terminology Criteria for Adverse Events (NCI, version 4.0, 2010), graded 0-4 [31]. 
Fig. 1 Extraoral PBM-the LED probe is applied flat against the patient's face and neck for $60 \mathrm{~s}$, at five treatment sites $\left(50 \mathrm{~mW} / \mathrm{cm}^{2} \times 60 \mathrm{~s}=3.0 \mathrm{~J} /\right.$ $\mathrm{cm}^{2}$ per location). Placebo/sham extraoral PBM protocol-an inactivated probe is applied flat against the patient's face and neck for $60 \mathrm{~s}$, at five same treatment sites as PBM protocol. Treatment sites: right face side (A), right neck (B), left face side $(\mathbf{C})$, left neck (D), and center face $(\mathbf{E}$ and $\mathbf{F})$.
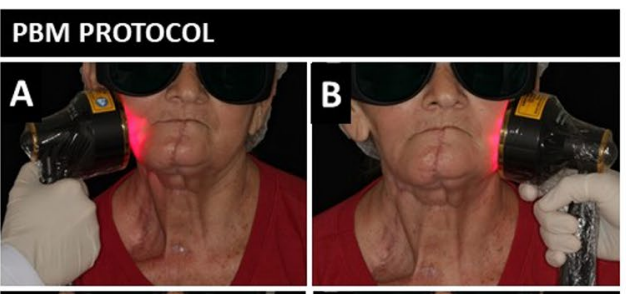

PLACEBO PROTOCOL
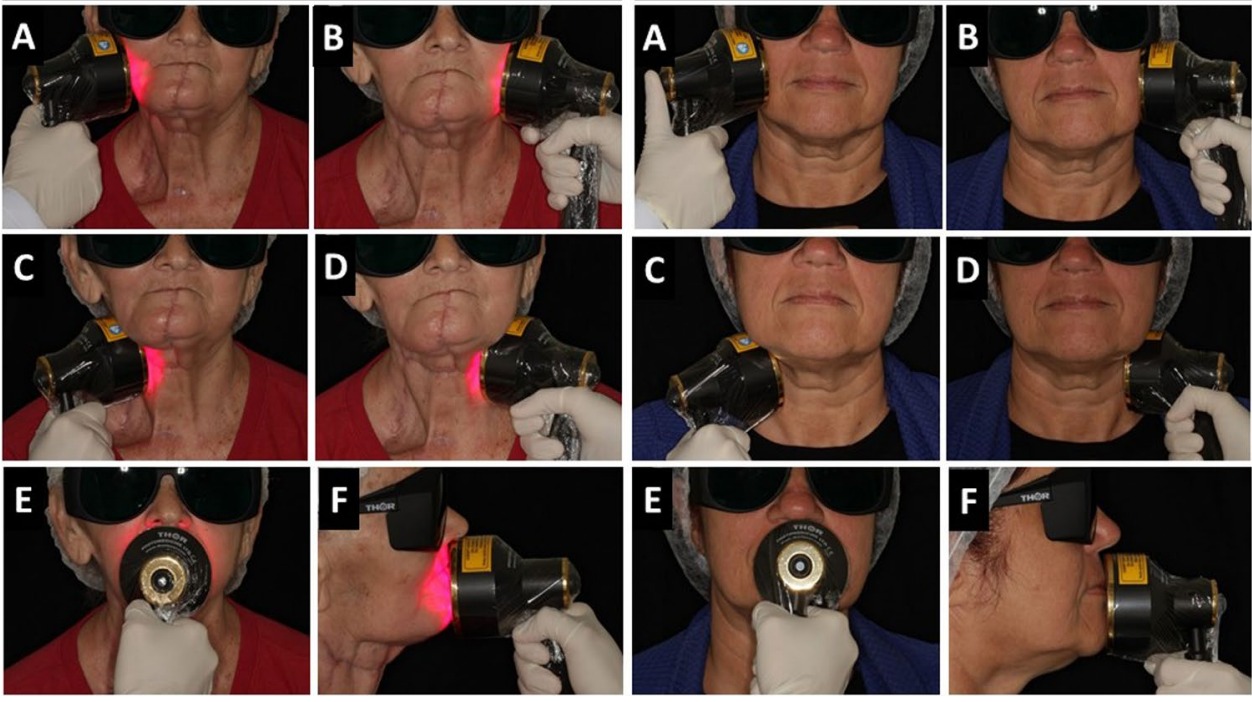

*PBM: photobiomodulation

\section{Pain and analgesia}

Pain was evaluated using a visual analogue scale (VAS) graded $0-10$. Medication used for OM analgesia was recorded weekly and classified by levels based on the pain scale and the WHO Analgesic Ladder: no analgesics, patients without pain related to OM; level 1, low level pain and non-opioid analgesics (VAS 1-3; paracetamol or dipyrone and/or ketoprofen or celecoxib); level 2, moderate pain and weak opioid (VAS 4-6; codeine or tramadol or dipyrone and/or ketoprofen); and level 3, severe pain and strong opioid (VAS 7-10; morphine or oxycodone + paracetamol or dipyrone and/or ketoprofen) $[12,14]$ and with or without adjuvants at each level.

\section{Anti-inflammatory prescriptions}

The prescription of anti-inflammatory agents for $\mathrm{OM}$ was also recorded weekly. All prescriptions were made by the medical team who were providing routine care and who were blinded to the study group allocation.

\section{Quality of life}

The University of Washington Quality of Life Questionnaire $(U W-Q O L v 4)$ validated for the Portuguese version [41] was completed before the first day of RT (D-1) and at the last day of RT (D35). The UW-QoL is composed of 12 objective questions of specific variables, ranging 0 to 100 , where 100 represent the best possible condition. The analysis was divided into physical and social-emotional function domains.

\section{Oncological outcomes}

After RT, patients were evaluated every 3 months for a total of 18 months. Evaluations were based on clinical examinations and medical information available in the electronic medical record to assess oncological outcomes. For cancer surveillance, overall survival (OS) rate, disease-free survival (DFS), the incidence of recurrences (local-regional and distant relapse rates), or new (second) primary tumors were the primary outcome measures [8].

\section{Statistical analysis}

Effectiveness was defined as the proportion of 30\% less severe OM in the PBM group compared to placebo, as proposed by the hypothesis of Legouté et al. [25]. Results of this interim analysis were expressed as mean values and percentages. Statistical significance rate of $5 \%(p \leq 0.05)$ was considered. Per protocol analysis of the data obtained from the present study, including Kaplan-Meier curve for 12-month period analysis of OS, was performed with GraphPad Prism 9.0. The Mann-Whitney test was used to analyze the $\mathrm{OM}$ overall incidence, pain and analgesia results, and QoL scores for group comparison. The chisquare test was used to compare incidence of severe OM, anti-inflammatory prescription, OM distribution, and OS. Finally, Wilcoxon signed-rank test was used to time comparison between single group QoL scores. 


\section{Research funding}

This trial had the financial support of the São Paulo Research Foundation (FAPESP) processes numbers 2018/02233-6 and 2018/23479-3, and the National Council for Scientific and Technological Development (CNPq).

\section{Results}

A total of 67 patients were randomized from June 2019 to November 2020. Twelve patients were excluded during RT due to noncompliance with RT $(n=1)$, RT interruption due to SARS-CoV-2 infection $(n=3)$, death before RT completed $(n=7)$, and OM grade 4 with medical request to discontinue the trial to receive therapeutic PBM ( $n=1$ /placebo group). Fifty-five participants who met inclusion criteria and completed the planned RT treatment were included in the clinical follow-up. The flow-chart and exclusion reasons are presented in Fig. 2.

Clinicopathological characteristics of the analyzed patients are summarized in Table 1. Patients from extraoral PBM and placebo groups had similar clinicopathological features; most of the patients were male (79.3\% vs. $84.6 \%)$, with a history of tobacco and alcohol use. The oropharynx was the most frequent primary tumor site for both groups, and CRT was the most common cancer treatment. There were no statistically significant differences in the clinicopathological characteristics between groups.

A total of 918 PBM sessions were performed for the PBM group and 832 sham sessions for the placebo group. There was no difference in the mean number of sessions

Fig. 2 Flowchart and outcomes

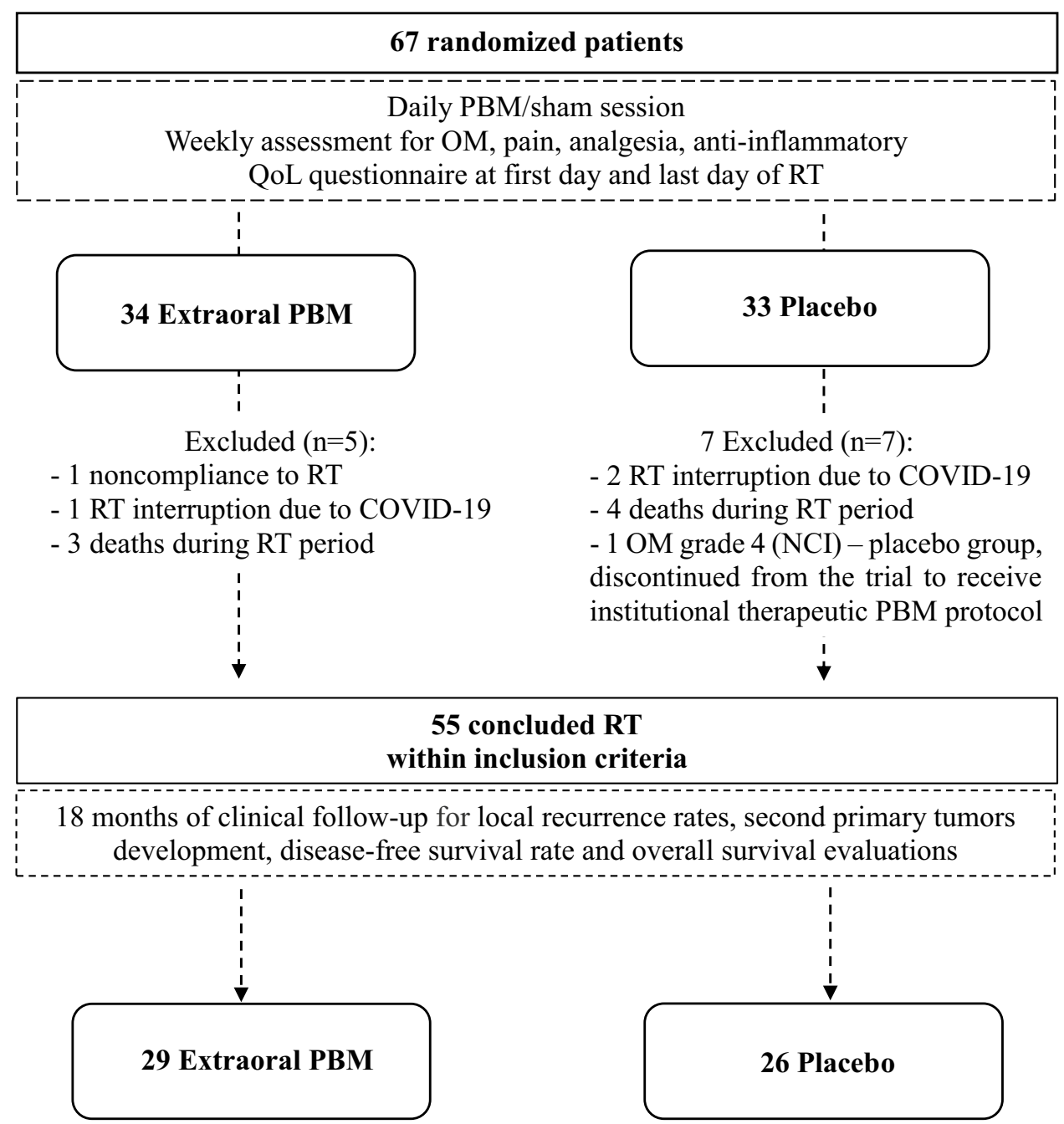

OM: Oral mucositis; RT: Radiotherapy; QoL: Quality of life; PBM: Photobiomodulation; NCI: National Cancer Institute. 
Table 1 Clinicopathological characteristics of included patients

\begin{tabular}{|c|c|c|c|c|c|}
\hline & PBM & & Placebo & & $p$-value* \\
\hline Patients $(n)$ & 29 & & 26 & & \\
\hline Gender & & & & & 0.73 \\
\hline Male & 23 & $(79.3 \%)$ & 22 & $(84.6 \%)$ & \\
\hline Female & 6 & $(20.7 \%)$ & 4 & $(15.4 \%)$ & \\
\hline Age (years) & & & & & 0.31 \\
\hline Mean \pm SD & 59.5 & $( \pm 8.1)$ & 62.1 & $( \pm 8.7)$ & \\
\hline Smoking status & & & & & 0.42 \\
\hline Never-smokers & 5 & $(17.3 \%)$ & 2 & $(7.7 \%)$ & \\
\hline Smokers & 3 & $(10.3 \%)$ & 6 & $(23.1 \%)$ & \\
\hline Smoking cessation & 21 & $(72.4 \%)$ & 18 & $(69.2 \%)$ & \\
\hline Smoking load (pack/years) & & & & & 0.32 \\
\hline Mean \pm SD & 46 & $( \pm 33.9)$ & 50,8 & $( \pm 30.8)$ & \\
\hline Alcohol consumption & & & & & 0.12 \\
\hline No & 9 & $(31.0 \%)$ & 5 & $(19.2 \%)$ & \\
\hline Yes-active use & 0 & $(0.0 \%)$ & 3 & $(11.5 \%)$ & \\
\hline Yes-alcohol withdrawal & 20 & $(68.1 \%)$ & 18 & $(69.3 \%)$ & \\
\hline \multicolumn{6}{|l|}{ Primary tumor site } \\
\hline Base of tongue & 5 & $(17.2 \%)$ & 4 & $(15.4 \%)$ & \\
\hline Tongue & 2 & $(6.9 \%)$ & 6 & $(23.1 \%)$ & \\
\hline Gingiva & 2 & $(6.9 \%)$ & 2 & $(7.7 \%)$ & \\
\hline Floor of mouth & 3 & $(10.4 \%)$ & 2 & $(7.7 \%)$ & \\
\hline Hard palate & 1 & $(3.4 \%)$ & 0 & $(0.0 \%)$ & \\
\hline Buccal mucosa & 3 & $(10.4 \%)$ & 0 & $(0.0 \%)$ & \\
\hline Palatine tonsil & 2 & $(6.9 \%)$ & 4 & $(15.4 \%)$ & \\
\hline Oropharynx with oral extension & 11 & $(37.9 \%)$ & 8 & $(30.7 \%)$ & \\
\hline Tumor stage & & & & & 0.23 \\
\hline III & 11 & $(37.9 \%)$ & 6 & $(23.1 \%)$ & \\
\hline IV & 18 & $(62.1 \%)$ & 20 & $(76.9 \%)$ & \\
\hline Histopathological differentiation & & & & & 0.92 \\
\hline Well-differentiated & 3 & $(10.3 \%)$ & 2 & $(7.7 \%)$ & \\
\hline Moderately differentiated & 15 & $(51.7 \%)$ & 12 & $(46.2 \%)$ & \\
\hline Poorly differentiated & 5 & $(17.3 \%)$ & 5 & $(19.2 \%)$ & \\
\hline Unknown & 6 & $(20.7 \%)$ & 7 & $(26.9 \%)$ & \\
\hline \multicolumn{6}{|l|}{ p16 status $* *$} \\
\hline Positive & 3 & $(23.1 \%)$ & 3 & $(25.0 \%)$ & \\
\hline Negative & 7 & $(53.8 \%)$ & 5 & $(41.7 \%)$ & \\
\hline Not available & 3 & $(23.1 \%)$ & 4 & $(33.3 \%)$ & \\
\hline Cancer treatment & & & & & 0.31 \\
\hline RT & 2 & $(6.9 \%)$ & 3 & $(11.5 \%)$ & \\
\hline RT + surgery & 6 & $(20.7 \%)$ & 8 & $(30.8 \%)$ & \\
\hline $\mathrm{CRT}+$ surgery & 6 & $(20.7 \%)$ & 5 & $(19.2 \%)$ & \\
\hline CRT & 15 & $(51.7 \%)$ & 10 & $(38.5 \%)$ & \\
\hline RT dose & & & & & 0.20 \\
\hline $60 \mathrm{~Gy}$ & 4 & $(13.8 \%)$ & 4 & $(15.4 \%)$ & \\
\hline $66 \mathrm{~Gy}$ & 10 & $(34.5 \%)$ & 14 & $(53.8 \%)$ & \\
\hline $70 \mathrm{~Gy}$ & 15 & $(51.7 \%)$ & 8 & $(30.8 \%)$ & \\
\hline PBM (sessions) & & & & & 0.38 \\
\hline Mean \pm SD & 32 & $( \pm 2.0)$ & 32 & $( \pm 1.7)$ & \\
\hline
\end{tabular}

$R T$ radiotherapy; $C R T$ chemoradiotherapy; $P B M$ photobiomodulation

*Mann-Whitney test for between-groups comparison (extraoral PBM vs. placebo)

${ }^{* *}$ p16 status was assessed for palatine tonsil and oropharynx tumors 
for both groups (32 sessions/patient; $p=0.38$ ). Excellent tolerance to PBM was reported by $54(98.1 \%)$ patients, while $1(1.9 \%)$ patient reported moderate tolerance associated with discomfort and nausea due to the smell of the disposable plastic film that covered the probe. No pain or adverse events were reported.

\section{Oral mucositis}

All patients experienced some grade of OM during RT (Fig. 3). The first occurrence was observed earlier in the placebo group (week 1) than the PBM group (week 2). Differences in the overall OM comparison were noted during week 1, in which no case of OM was observed in the PBM compared to the OM incidence of $19 \%$ in the placebo group $(p=0.014)$ and during week 2 , where OM incidence was $55 \%$ for the PBM group in comparison with $85 \%$ for the placebo $(p=0.009)$. Comparison over the time of RT showed a later OM onset for the PBM group. During week 3, 100\% of the placebo group experienced some grade of $\mathrm{OM}$, and the same results were observed at week 6 for the PBM group.

Incidence of severe $\mathrm{OM}$ (grade $\geq 3$ ) was higher in the placebo group during all study periods evaluated, with the exception of the last week of RT, where PBM showed 52\% of grade 3 OM vs. $41 \%$ at the placebo group $(p=0.469)$. There was no difference in terms of percentage ( $\geq 30 \%$ ratio of grade $\geq 3$ ) for severe OM incidence between groups in any period of evaluation, including the last week of treatment $(p=0.447)$.

For the PBM group, the OM incidence was associated with oral mucosal sites distant from the direct contact with the extraoral probe. At the last week of treatment, oropharynx (16\%), border of the tongue (14\%), and retromolar trigone (14\%) were the most affected sites for the PBM group. The results for the placebo group were border of the tongue (15\%), oropharynx (14\%), and buccal mucosa (14\%), an area with direct contact with the extraoral probe (Supplementary Fig. 1).

\section{Pain and analgesia}

Pain evaluations are shown in Fig. 3. During most of the periods of assessment, lower mean pain score was observed for the PBM group; the highest mean score was 2.8 during week 5 of RT. Moderate pain score (VAS, 3-7) was observed in the placebo group during week 6 (3.3) and week 7 (4.5), representing the highest mean level of pain in the placebo group during the observation period. Significant statistical difference was observed at week 7 with mean pain score of 2.1 for the PBM group vs. 4.5 for the placebo group $(p=0.009)$, the highest mean pain score observed in the study.
During all periods of evaluation, the PBM group had a lower percentage of patients that required analgesics (Table 2). During week 3, 48.2\% of PBM vs. $76.9 \%$ of placebo required analgesics for pain relief, and while no patient in the PBM group used opioids, $2(7.7 \%)(p=0.009)$. Similar results were observed during week 7 where $48 \%$ of PBM patients vs. $86.4 \%$ of placebo were using any analgesic for OM-related pain relief, and a higher prevalence of opioids analgesic use was observed $4.0 \%$ of PBM vs. $27.3 \%$ of placebo patients $(p=0.02)$.

\section{Anti-inflammatory prescription}

The numbers of anti-inflammatory prescriptions were higher in the placebo group (Fig. 3). At week 4 of RT, the maximum number of prescriptions was observed for both groups, with a higher percentage for placebo (34.6\%) in comparison with the PBM group (20.7\%) $(p=0.5879)$. At week 5, a difference of anti-inflammatory prescription between groups was seen, with $30.8 \%$ for the placebo and $6.9 \%$ for the PBM group $(p=0.0346)$.

\section{Quality of life}

The QoL assessments are presented in Fig. 3. The general UW-QoL score at D1 and D35 for the PBM group was 910 and 687, respectively, while for the placebo group were 868 and 607, respectively. Statistically significant results were found at D35 for general QoL for between groups comparison $(p=0.0390)$.

At D35, the physical QoL mean score was lower for the placebo group (258 vs. 279 for the PBM group $(p=0.1330)$ ), similar to the social-emotional QoL with scores of 348 for the placebo group vs. 408 for the PBM group $(p=0.0034)$.

In terms of treatment period comparison (D1 vs. D35), a negative impact of RT on patients QoL was observed at D35 for both groups and in all QoL outcomes. A statistically significant difference in general and physical outcomes for both placebo and PBM groups were observed $(p>0.0001)$ and social-emotional outcome for placebo group. The socialemotional QoL outcome for PBM group was an exception $(p=0.1553)$.

\section{Oncological outcomes}

In 1 year of follow-up, no local or systemic adverse events due to the PBM were observed. One local recurrence was recorded 6 months follow-up in the placebo group. No second primary tumors were observed. An interim analysis of the OS with the mean follow-up period of 12 months was possible, and a slight tendency for better overall survival was observed in the PBM group (74.0 vs. $68.7 \% ; p=0.889$; 


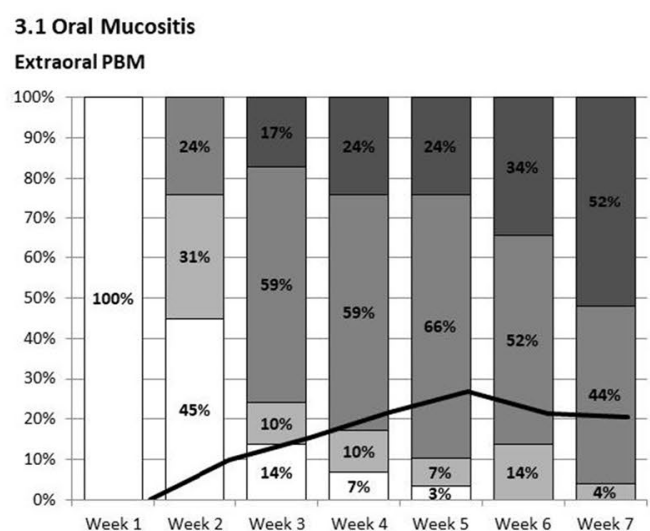

Placebo

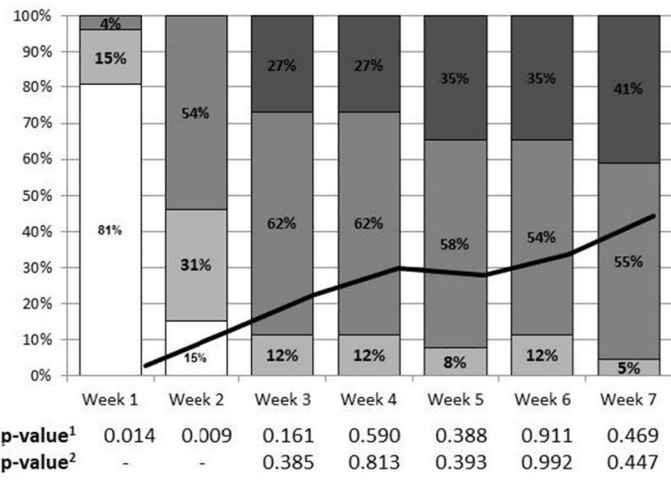

p-value 1: Mann-Whitney test for between-groups overall OM comparison (Extraoral PBM vs Placebo)

p-value ${ }^{2}$ : Chi-square test for between-groups severe OM comparison (Extraoral PBM vs Placebo)

\subsection{Pain Score (VAS)}

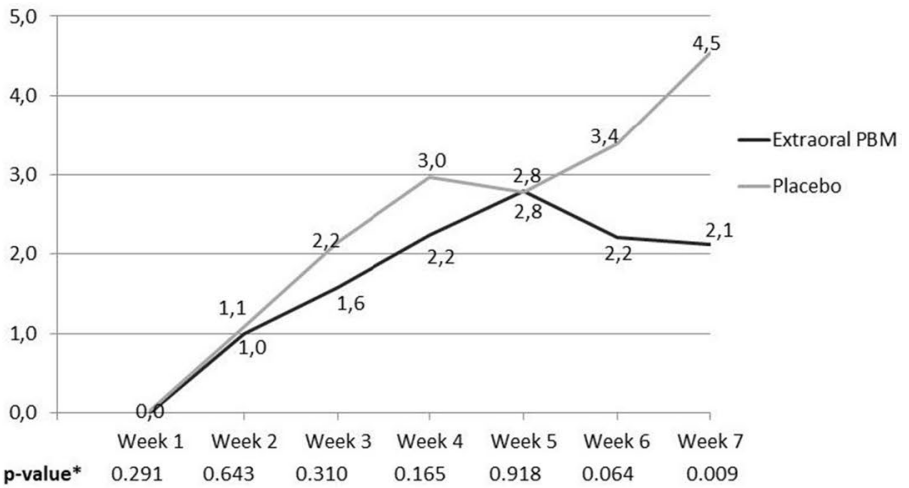

* Mann-Whitney test for between-groups comparison (Extraoral PBM vs. placebo)

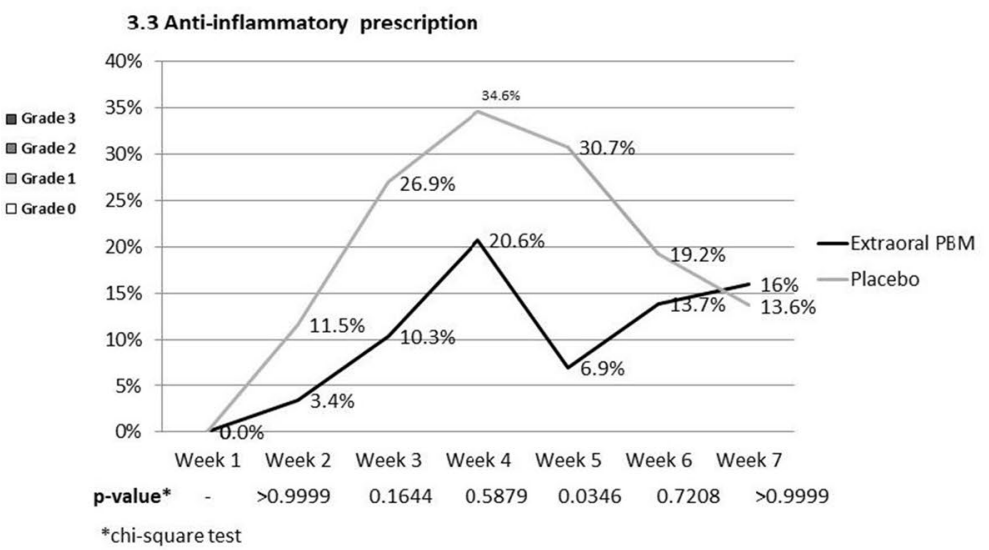

3.4 Quality of Life (QoL)
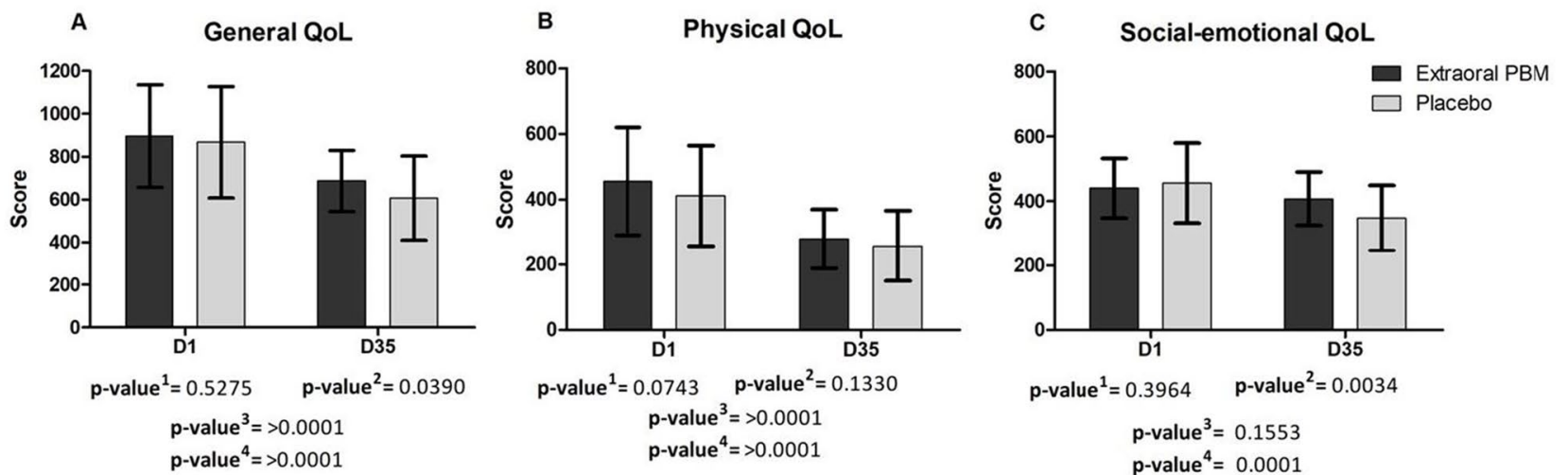

1: Mann-Whitney test for between-groups comparison in D1 evaluation time (Extraoral PBM vs. Placebo)

2: Mann-Whitney test for between-groups comparison in D35 evaluation time (Extraoral PBM vs. Placebo)

3: Wilcoxon paired signed rank for between evaluation time comparison (Extraoral PBM group - D1 vs. D35)

${ }^{4}$ : Wilcoxon paired signed rank for between evaluation time comparison (Placebo group - D1 vs. D35)

Fig. 3 Clinical assessments. 3.1: Oral Mucositis-Weekly oral mucositis assessment according to the National Cancer Institute (NCI, version 4.0; 2010). Bars represent percent of cases in each oral mucositis grade and continuous lines represent mean values for each stage (score range from 0 to 4); 3.2 Pain Score-Oral mucositis associated pain score (visual analogue scale - VAS); 3.3: Anti-inflamma- tory prescription at the different weeks of RT treatment; 3.4 Quality of life-Graphs comparing mean $( \pm \mathrm{SD})$ University of Washington Quality of Life Questionnaire (UW-QoL v4) score at baseline (D1) and final session of radiotherapy (D35). Graph A, general QoL; graph B, physical QoL; graph C, social-emotional QoL 


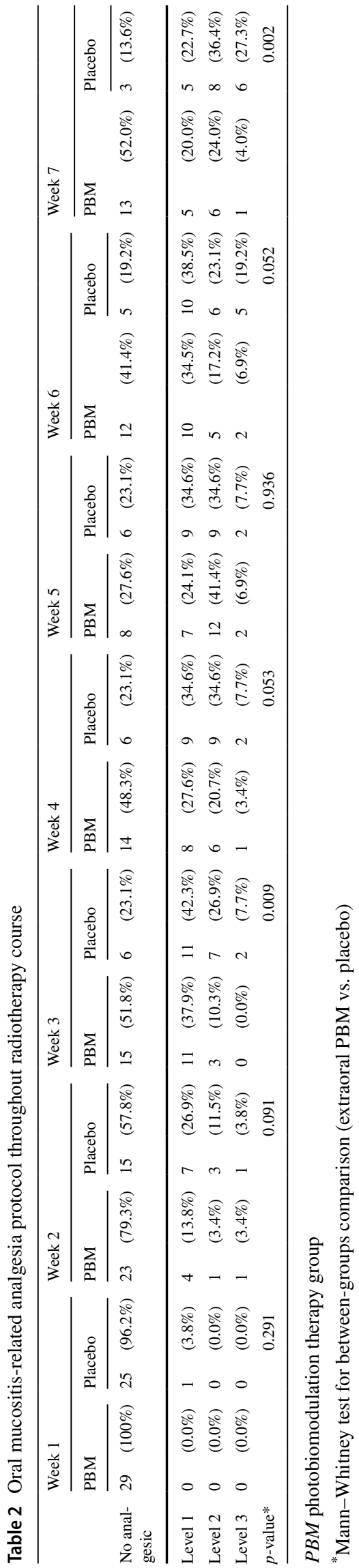

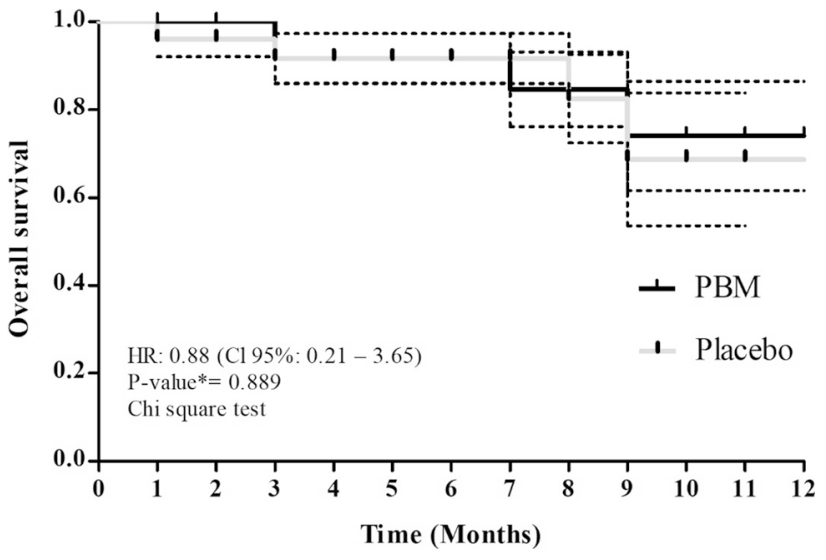

PBM : photobiomodulation

Fig. 4 Interim analysis of the overall survival with the follow-up period of 12 months

HR 0.88; CI 95\% 0.21-3.65) (Fig. 4). These data will be updated after a total follow-up period of 18 months after the last patient enrollment.

\section{Discussion}

We evaluated the effects of a prophylactic extraoral PBM in the outcomes of RT-induced OM and oncological outcomes. The demographic characteristics of the included patients in this interim analysis were similar to those presented in the literature, patients with advanced OOPSCC, mostly males, with history of tobacco and alcohol use [2, 17, 20, 25]. Additionally, the oncological treatment reflected the standard of care from international cancer centers, based on a multimodal approach, associated with a better prognosis, but also with an increase of acute side effects, particularly OM [2].

In our study, a delay in the development of OM for the PBM group, along with a difference on severity duration due to later OM onset, reinforces the prophylactic effect of PBM. However, there was a high incidence of grade 3 OM for both groups during the last week of treatment. While there is robust evidence of the effectiveness of PBM in OM [43], different PBM effectiveness results can be attributed to many factors including PBM parameters, oncological treatment regimen, and patient's characteristics [10, 11, 19, 25]. One of the challenges when comparing PBM results between studies is the heterogeneity of PBM protocols and parameters used [5, 8, 10, 17, 44]. Few studies have evaluated the effectiveness of extraoral PBM for OM [16, 18, 41], due to the lack of evidence and the lack of validated protocols for extraoral PBM for OM [21]. 
During the last week of RT, we observed severe OM primarily in the oropharynx, and posterolateral border of the tongue. The oropharynx was the most frequent primary tumor site for both groups, with the primary radiation dose the area and greater difficulty in OM management. Also, these areas with greater OM grade 3 were distant from the extraoral light surface, and the literature shows that light delivery to target tissue is affected by its distance from the light source [5, 10, 22, 44, 45]. For extraoral PBM, tissues with greater energy delivered include the buccal mucosa, the vestibule, and the oral surfaces of the lips $[5,10,44,45]$.

PBM effectiveness on severe OM control may also be due to insufficient PBM parameters, and adjustments in the extraoral PBM protocol need to be optimized with the goal of achieving greater efficacy. The use of extraoral application plus intraoral delivery on selected high-risk oral regions per radiation treatment plan may enhance compliance and reduce time for light application in the clinical setting. Additional studies are warranted. Furthermore, the evaluation of site-specific patterns of OM may improve the development of PBM protocols [4, 12]. It is important to highlight that extraoral PBM is considered to be a simple, well tolerated, and easily applied intervention.

In our study, patients from the PBM group experienced less severe pain associated with OM, lower mean pain score during RT with reduced opioid use. Important differences in pain assessment and analgesics between PBM and placebo were observed to be greatest during the last week of RT. PBM is known to be associated with pain reduction and thus may lead to reduced use of opioid analgesics [22, 26, 33, 38, 45]. Similar studies, Antunes et al. [26] and Gautam et al. [1] reported significantly less severe oral pain scores for PBM-treated patients compared to placebo, in addition to reduced opioid use during RT.

Higher prescriptions of anti-inflammatory agents were observed in the placebo group, which may also have influenced the OM severity incidence. Although no guideline supports the use of systemic anti-inflammatory agents to manage OM, inflammation is considered to be an important major effect of RT-induced OM and anti-inflammatory inhibition is a potential treatment strategy in this context $[16,18]$.

Oral and oropharyngeal cancer is associated with reduced QoL due to the effects of primary tumor and treatment side effects impairing patient's daily functional and self-image [3, 17]. Worsening levels of general QoL were observed at the end of the treatment, as reported in previously published studies [3, 15, 26, 33]. The variability of QoL is directly associated with cancer treatment toxicities' alterations in swallowing, chewing, saliva changes, taste, and especially OM-related pain [3]. Our study shows better social-emotional QoL in those treated with PBM, which could be explained by the positive impact in OM symptom attenuation specifically decreased pain levels $[15,26]$.

It is imperative that an intervention used to support cancer patients during therapy does not adversely affect tumor behavior, or tumor response to treatment $[5,25,28$, 37, 39]. Data about PBM impact on tumor activity and oncological treatment response based on in vitro studies are conflicting. Contradictory results may be correlated to the variation of PBM parameters, tumor cell lines, and tumor genomic heterogeneity between studies [13, 21, 39]. Current literature indicates that any in vitro experiment assessing the effect of PBM should not be considered representative of what happens in the clinical care. Based on the existing data, confirmation of the safety of PBM in the management of $\mathrm{OM}$ is important to be examined in prospective randomized controlled clinical trials in oral and oropharynx tumors $[6,8]$. Our evaluation of tumor outcomes was not adversely affected by PBM.

No significant adverse side effects were noted in the present study in the setting of oral and oropharynx cancer patients submitted to PBM during RT. This is in agreement with the current literature $[1,8,10,13,17,25,26,30]$. Furthermore, no relevant negative effect of PBM on tumor biology was demonstrated, also in agreement with other similar studies [8, 13, 25, 26, 34]. No differences in OS were seen in the current study in PBM vs. placebo groups. Additional data will be available upon the final analysis of 18 months of follow-up. As PBM mechanisms continue to be studied, the effects of different parameters on tumor heterogeneity will add information based on solid science $[6,8]$.

\section{Limitations of the study}

The present study is a planned interim analysis of an ongoing clinical trial and results could change at completion of the trial and enlargement of the study sample.

\section{Conclusions}

This prospective double-blind randomized clinical trial assessed clinical and oncological outcomes of prophylactic extraoral PBM in radiation-induced OM in OOPSCC patients. Extraoral PBM was well tolerated and did not cause any significant adverse effects. This planned interim analysis suggests the indication of prophylactic PBM to prevent the early onset of OM, to reduce pain levels and reduce the need of analgesics and anti-inflammatory medications in OOPSCC patients submitted to RT. Furthermore, no impact on tumor behavior or control and survival outcomes were seen, within the limits of the interim results of this clinical trial. 
Supplementary Information The online version contains supplementary material available at https://doi.org/10.1007/s00520-021-06625-8.

Author contribution Elisa Kauark-Fontes: study design, data acquisition, data analysis and interpretation, manuscript preparation, and editing.

Cesar Augusto Migliorati: study concept, manuscript preparation, and review.

Joel B Epstein: data analysis and interpretation, manuscript preparation, and review.

Nathaniel Simon Treister: study design, quality control of data, manuscript review.

Carolina Guimarães Bonfim Alves: data acquisition, data analysis and interpretation, statistical analysis.

Karina Morais Faria: data acquisition, manuscript preparation, and review.

Natalia Rangel Palmier: study design, data acquisition, manuscript review.

Leticia Rodrigues-Oliveira: data acquisiton, manuscript editing, and review.

Mariana de Pauli Paglioni: study design, quality control of data.

Luiz Alcino Monteiro Gueiros: data analysis, statistical analysis, manuscript review.

Karina G M da Conceição Vasconcelos: quality control of data and manuscript review.

Gilberto de Castro Jr: study concept, study design, quality of data.

Adriana Franco Paes Leme: study concepts, manuscript review.

Marcio Ajudarte Lopes: quality contol of data, manuscript review.

Ana Carolina Prado-Ribeiro: study concept and design, data acquisition, manuscript editing.

Thaís Bianca Brandão: study concept and design, data analysis and interpretation, manuscript review.

Alan Roger Santos-Silva: study concept and design, data analysis and interpretation, manuscript preparation, and review.

Funding The authors gratefully acknowledge the financial support of the São Paulo Research Foundation (FAPESP) numbers 2018/02233-6 and 2018/23479-3; and the National Council for Scientific and Technological Development (CNPq). Alan Roger Santos-Silva is a CNPq research grantee.

Data availability The authors confirm that the data supporting the findings of the study are available within the article and supplementary materials.

\section{Code availability N/A.}

\section{Declarations}

Ethics approval Brazil National Human Research Ethics Committee (CAAE: 21648819.9.0000.5418).

Consent to participate All participants included in the study provided informed consent.

Consent for publication All participants included in the study provided informed consent.

Conflict of interest The authors declare no competing interests.

\section{References}

1. Antunes HS, Herchenhorn D, Small IA, Araújo CM, Viégas CM, Cabral E, Rampini MP, Rodrigues PC, Silva TG, Ferreira EM, Dias FL, Ferreira CG (2013) Phase III trial of low-level laser therapy to prevent oral mucositis in head and neck cancer patients treated with concurrent chemoradiation. Radiother Oncol 109:297-302

2. Antunes HS, Herchenhorn D, Small IA, Araujo CMM, Viégas CMP, Ramos GA et al (2014) Long-term survival of a randomized phase III trial of head and neck cancer patients receiving concurrent chemoradiation therapy with or without low-level laser therapy (LLLT) to prevent oral mucositis. Oral O 71(11):15

3. Ariyawardana A, Cheng KKF, Kandwal A, Tilly V, Al-Azri AR, Galiti D et al (2019) Systematic review of anti-inflammatory agents for the management of oral mucositis in cancer patients and clinical practice guidelines. Support Care Cancer 27:3985-3995

4. Arora H, Pai KM, Maiya A, Vidyasagar MS, Rajeev A (2008) Efficacy of He-Ne Laser in the prevention and treatment of radiotherapy-induced oral mucositis in oral cancer patients. Oral Surg Oral Med Oral Pathol Oral Radiol Endod 105:180-186

5. Bensadoun RJ (2018) Photobiomodulation or low-level laser therapy in the management of cancer therapy-induced mucositis, dermatitis and lymphedema. Curr Opin Oncol 30:226-232

6. Bensadoun RJ, Epstein JB, Nair RG, Barasch A, Raber-Durlacher JE, Migliorati C et al (2020) World Association for Laser Therapy (WALT) Safety and efficacy of photobiomodulation therapy in oncology: a systematic review. Cancer Med 9(8279):300

7. Bjordal JM, Bensadoun RJ, Tuner J, Frigo L, Gjerde K, LopesMartins RA (2011) A systematic review with meta-analysis of the effect of low-level laser therapy (LLLT) in cancer therapy-induced oral mucositis. Support Care Cancer 19:1069-1077

8. Brandao TB, Morais-Faria K, Ribeiro ACP, Rivera C, Salvajoli JV, Lopes MA et al (2018) Locally advanced oral squamous cell carcinoma patients treated with photobiomodulation for prevention of oral mucositis: retrospective outcomes and safety analyses. Support Care Cancer 26:2417-2423

9. Brierley JD, Gospodarowicz MK, Wittekind CW. 2017 International Union Against Cancer (UICC). TNM Classification of Malignant Tumours, Eighth Edition. Wiley

10. Cooper JS, Pajak TF, Forastiere AA, Jacobs J, Campbell BH, Saxman SB et al (2004) For the Radiation Therapy Oncology Group 9501/Intergroup Postoperative concurrent radiotherapy and chemotherapy for high-risk squamous-cell carcinoma of head and neck. Engl J Med 350:1937-44

11. De Lima AG, Villar RC, De Castro G Jr, Antequera R, Gil E, Rosalmeida MC et al (2012) Oral mucositis prevention by lowlevel laser therapy in head-and-neck cancer patients undergoing concurrent chemoradiotherapy: a phase III randomized study. Int J Radiation Oncology Biol Phys 82:270-275

12. de Pauli PM, Faria KM, Palmier NR, Prado-Ribeiro AC, Dias RBE, Pinto HG et al (2021) Patterns of oral mucositis in advanced oral squamous cell carcinoma patients managed with prophylactic photobiomodulation therapy-insights for future protocol development. Lasers Med Sci 36:429-436

13. Elad S, Arany P, Bensadoun RJ, Epstein JB, BaraschRaber-Durlacher A (2018) Photobiomodulation therapy in the management of oral mucositis: search for the optimal clinical treatment parameters. Support Care Cancer 26(3319):321

14. Ferreira KASL, Kimura M, Teixeira MJ (2006) The WHO analgesic ladder for cancer pain control, twenty years of use. How much pain relief does one get from using it? Support Care Cancer 14:1086-1093

15. Franco P, Martini S, Di Muzio J et al (2017) Prospective assessment of oral mucositis and its impact on quality of life and 
patient-reported outcomes during radiotherapy for head and neck cancer. Med Oncol 34:81

16. Gautam AP, Fernandes DJ, Vidyasagar MS, Maiya AG, Guddattu V (2015) Low level laser therapy against radiation induced oral mucositis in elderly head and neck cancer patients-a randomized placebo controlled trial. J Photochem Photobiol B 144:51-56

17. Genot-Klastersky MT, Klastersky J, Awada F, Awada A, Crombez $\mathrm{P}$, Martinez MD et al (2008) The use of low-energy laser (LEL) for the prevention of chemotherapy- and/or radiotherapy-induced oral mucositis in cancer patients: results from two prospective studies. Support Care Cancer 16:1381-1387

18. Gruber S, Bozsaky E, Roitinger E, Schwarz K, Schmidt M, Dörr W (2017) Early inflammatory changes in radiation-induced oral mucositis: effect of pentoxifylline in a mouse model. Strahlenther Onkol 193:499-507

19. Guedes CDCFV, de Freitas Filho SAJ, de Faria PR, Loyola AM, Sabino-Silva R, Cardoso SV (2018) Variation of energy in photobiomodulation for the control of radiotherapy-induced oral mucositis: a clinical study in head and neck cancer patients. Int $\mathrm{J}$ Dent 4579279:1-6

20. Güneri P, Epstein JB (2014) Late stage diagnosis of oral cancer: components and possible solutions. Oral Oncology 50(12):1131-6

21. Hamblin MR, Nelson ST, Strahan JR (2018) Photobiomodulation and cancer: what is the truth? Photomed Laser Surg 36:241-245

22. Hodgson BD, Margolis DM, Salzman DE, Eastwood D, Tarima S, Williams LD et al (2012) Amelioration of oral mucositis pain by NASA near-infrared light emitting diodes in bone marrow transplant patients. Support Care Cancer 20:1405-1415

23. Hong CHL, Gueiros LA, Fulton JS, Cheng KKF, Kandwal A, Galiti D et al (2019) Mucositis Study Group of the Multinational Association of Supportive Care in Cancer/International Society for Oral Oncology (MASCC/ISOO). Systematic review of basic oral care for the management of oral mucositis in cancer patients and clinical practice guidelines. Support Care Cancer 27:3949 67

24. Kauark-Fontes E, Rodrigues-Oliveira L, Epstein JB, Faria KM, Araújo ALD, Gueiros LAM et al (2021) Cost-effectiveness of photobiomodulation therapy for the prevention and management of cancer treatment toxicities: a systematic review. Support Care Cancer 29(6):2875-2884. https://doi.org/10.1007/ s00520-020-05949-1

25. Legouté F, Bensadoun RJ, Seegers V, Pointreau Y, Caron D, Lang $P$ et al (2019) Low-level laser therapy in treatment of chemoradiotherapy-induced mucositis in head and neck cancer: results of a randomised, triple blind, multicentre phase III trial. Radiat Oncol $14: 83$

26. Lima AG, Antequera R, Peres MPSM, Snitcosky IML, Federico MHH, Villar RC (2010) Efficacy of low-level laser therapy and aluminum hydroxide in patients with chemotherapy and radiotherapy-induced oral mucositis. Braz Dent J 21:186-192

27. Maria OM, Eliopoulos N, Muanza T (2017) Radiation-induced oral mucositis. Front Oncol 7:89

28. Martins AL, Morais MO, de Sousa-Neto SS, Jesus APG, Nogueira TE, Valadares MC et al (2021) Photobiomodulation reduces the impact of radiotherapy on oral health-related quality of life due to mucositis-related symptoms in head and neck cancer patients. Lasers Med Sci 36:903-912

29. Morais KF, Gomes-Silva W, Kauark-Fontes E, Bonfim-Alves CG, Kowalski LP, Prado-Ribeiro AC et al (2020) Impact of pandemic COVID-19 outbreak on oral mucositis preventive and treatment protocols: new perspectives for extraoral photobiomodulation therapy. Support Care Cancer 28:4545-4548

30. Myakishev-Rempel M, Stadler I, Brondon P, Axe DR, Friedman M, Nardia FB et al (2012) A preliminary study of the safety of red light phototherapy of tissues harboring cancer 30:551-558
31. National Cancer Institute (2010). Common terminology criteria for adverse events (CTCAE) version 4.0 published: May 28, 2009 (v4.03: June14, 2010). Downloaded from http://evs.nci.nih.gov/ ftp1/CTCAE/CTCAE_4.03_2010-06-14_QuickReference_5x7. pdf on Mar 30, 2011. Last accessed in 27 of April of 2021.

32. Oncological safety of intraoral and extraoral photobiomodulation in patients with oral and oropharyngeal squamous cell carcinoma. Ensaiosclinicos.gov. https://ensaiosclinicos.gov.br/rg/ RBR-4w4swx - Accessed 10 Jan\&nbsp;2021

33. Paglioni MP, Alves CGB, Fontes EK, Lopes MA, Ribeiro ACP, Brandão TB et al (2019) Is photobiomodulation therapy effective in reducing pain caused by toxicities related to head and neck cancer treatment? A systematic review Support Care Cancer 27:4043-4054

34. Paglioni MP, Araújo ALD, Arboleda LPA, Palmier NR, Fonseca JM, Gomes-Silva W et al (2019) Tumor safety and side effects of photobiomodulation therapy used for prevention and management of cancer treatment toxicities. A systematic review Oral Oncol 93:21-28

35. Schulz KF, Altman DG, Moher D (2010) CONSORT 2010 Statement: updated guidelines for reporting parallel group randomised trials. BMJ 340:c332-c432

36. Sonis ST (2011) Oral mucositis. Anticancer Drugs 22:607-612

37. Sonis S (2019) Could the impact of photobiomodulation on tumor response to radiation be effected by tumor heterogeneity? Support Care Cancer 28:423-424

38. Sonis ST, Eilers JP, Epstein JB, LeVeque FG, Liggett WH Jr, Mulagha MT et al (1999) Mucositis Study Group Validation of a new scoring system for the assessment of clinical trial research of oral mucositis induced by radiation or chemotherapy. Cancer 85(2103): 13

39. Sonis TS, Hashemi S, Epstein JB, Nair RG, Raber-Dulacher JE (2016) Could the biological robustness of low level laser therapy (photobiomodulation) impact its use in the management of mucositis in head and neck cancer patients. Oral Oncol 54:7-14

40. Treister NS, London WB, Guo D, Malsh M, Verrill K, Brewer $\mathrm{J}$ et al (2016) A feasibility study evaluating extraoral photobiomodulation therapy for prevention mucositis in pediatric hematopoietic cell transplantation. Photomed Laser Surg 34:178-184

41. Vartanian JG, Carvalho AL, Yueh B, Furia CLB, Toyota J, McDowell A et al (2006) Brazilian-Portuguese validation of the University of Washington quality of life questionnaire for patients with head and neck. Head Neck 28:1115-1121

42. Villa A, Sonis ST (2015) Mucositis: pathobiology and management. Curr Opin Oncol 27:159-164

43. Zadik Y, Arany PR, Fregnani ER, Bossi P, Antunes HS, Bensadoun $\mathrm{R}$ et al (2019) Systematic review of photobiomodulation for the management of oral mucositis in cancer patients and clinical practice guidelines. Support Care Cancer 27:3969-3983

44. Zecha JAEM, Raber-Durlacher JE, Nair RG, Epstein JB, Sonis ST, Elad S et al (2016) Low level laser therapy/photobiomodulation in the management of side effects of chemoradiation therapy in head and neck cancer: part 1: mechanisms of action, dosimetric, and safety considerations. Support Care Cancer 24:2781-2792

45. Zecha JAEM, Raber-Durlacher JE, Nair RG, Epstein JB, Elad S, Hamblin MR et al (2016) Low-level laser therapy/photobiomodulation in the management of side effects of chemoradiation therapy in head and neck cancer: part 2: proposed applications and treatment protocols. Support Care Cancer 24:2793-2805

Publisher's note Springer Nature remains neutral with regard to jurisdictional claims in published maps and institutional affiliations. 


\section{Authors and Affiliations}

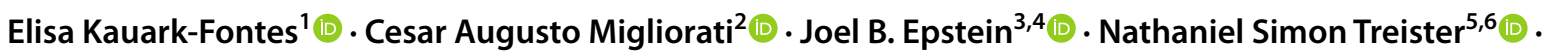

Carolina Guimarães Bonfim Alves ${ }^{1,7}(\mathbb{1}) \cdot$ Karina Morais Faria ${ }^{7}(\mathbb{1}) \cdot$ Natalia Rangel Palmier ${ }^{1,7}$ (1)

Leticia Rodrigues-Oliveira ${ }^{1}$ (D) Mariana de Pauli Paglioni ${ }^{1}$ (D) . Luiz Alcino Monteiro Gueiros ${ }^{8}$ (D) .

Karina G. M. da Conceição Vasconcelos ${ }^{9,10}$ (1) . Gilberto de Castro Jr ${ }^{11}\left(\right.$ (1) . Adriana Franco Paes Leme ${ }^{12}$ (1) .

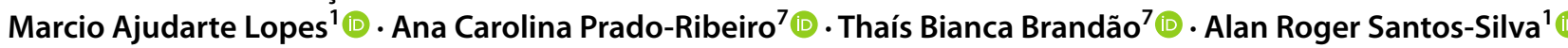

1 Oral Diagnosis Department, Piracicaba Dental School, University of Campinas (UNICAMP), Av. Limeira, 901, Bairro Areão, Piracicaba, SP 13414-903, Brazil

2 College of Dentistry, University of Florida, Gainesville, FL, USA

3 Samuel Oschin Comprehensive Cancer Center, Cedars-Sinai Health System, Los Angeles, CA, USA

4 City of Hope Comprehensive Cancer Center, Duarte, CA, USA

5 Division of Oral Medicine and Dentistry, Brigham and Women's Hospital, Boston, MA, USA

6 Department of Oral Medicine, Infection and Immunity, Harvard School of Dental Medicine, Boston, MA, USA
Dental Oncology Service, Instituto Do Cancer Do Estado de São Paulo (ICESP-FMUSP), São Paulo, SP, Brazil

8 Clinical and Preventive Dentistry Department, Universidade Federal de Pernambuco, Recife, SP, Brazil

9 Service of Radiation Oncology, Instituto Do Câncer Do Estado de São Paulo (ICESP), Faculdade de Medicina da Universidade de São Paulo (FMUSP), São Paulo, SP, Brazil

10 Radiotherapy D’Or, São Paulo, SP, Brazil

11 Department of Medical Oncology, Instituto Do Cancer Do Estado de São Paulo (ICESP), São Paulo, SP, Brazil

12 Brazilian Biosciences National Laboratory (LNBio), Brazilian Center for Research in Energy and Materials (CNPEM), Campinas, São Paulo, Brazil 